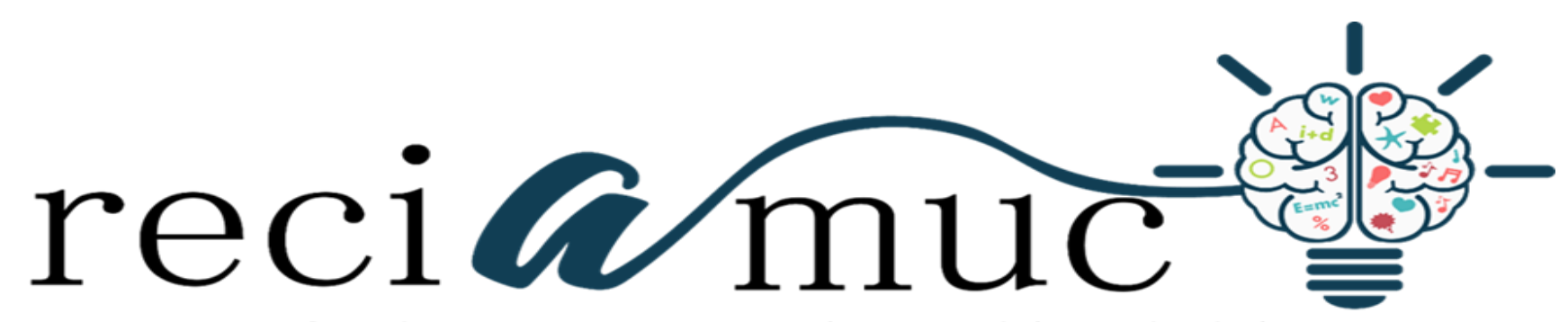

Revista cientifica de investígación actualización del mundo de las ciencias

Santiago Darío Freire de la A ${ }^{\text {a; }}$ Sofía Teresa Valdez Castro ${ }^{\text {b; }}$ Antonella Fanny Montenegro Villavicencio ${ }^{c}$; Lucía Andrea Jiménez Rivera ${ }^{d}$

Cardiopatía reumática diagnóstico y tratamiento

Rheumatic heart disease diagnosis and treatment

Revista Científica de Investigación actualización del mundo de las Ciencias. Vol. 3 núm., 4, octubre: 2588-0748, 2019, pp. 41-55

DOI: $10.26820 /$ reciamис/3.(4).octubre.2019.41-55

URL: http://reciamuc.com/index.php/RECIAMUC/article/view/387

Código UNESCO: 3205 Medicina Interna

Tipo de Investigación: Artículo de Revisión

(C) RECIAMUC; Editorial Saberes del Conocimiento, 2019

Recibido: $15 / 05 / 2019$

Aceptado: 07/08/2019

Publicado: 01/10/2019

Correspondencia: $\underline{\text { sant freire@ @otmail.com }}$
a. Médico; Investigador Independiente; Guayaquil, Ecuador; sant freire@ @otmail.com
b. Médico; Investigador Independiente; Guayaquil, Ecuador; Sophiavaldezc_91@ @otmail.com
c. Médico; Investigador Independiente; Guayaquil, Ecuador; anto_montenegro@ hotmail.com
d. Médico; Investigador Independiente; Guayaquil, Ecuador; jlucir@ hotmail.com 


\section{Cardiopatía reumática diagnóstico y tratamiento}

Vol. 3, núm. 4., (2019)

Santiago Darío Freire de la A; Sofía Teresa Valdez Castro; Antonella Fanny Montenegro Villavicencio; Lucía Andrea Jiménez Rivera

\section{RESUMEN}

Cardiopatía reumática es considerada como uno de los flagelos cardiovasculares más graves del siglo pasado. La enfermedad fue casi erradicada en el mundo desarrollado en la década de 1980, gracias a las mejoras en las condiciones de vida y la introducción de la penicilina. A nivel mundial, las enfermedades cardiovasculares son la principal causa de mortalidad, registrando en 2013 más de 17.3 millones de muertes, una cifra que para el año 2030 se cree aumente a más de 23.6 millones. Sobre el Ecuador, la OMS mediante un reporte ha informado que se ha evidenciado un incremento en la morbilidad y mortalidad de las enfermedades crónicas no transmisibles, así como también se deja ver que las enfermedades infecciosas aún son persistentes (entre esas, evidentemente figura la cardiopatía reumática), no obstante, mediante otro reporte previo habían estimado que para 2025 la tendencia por muerte prematura por enfermedades cardiovasculares disminuirían significativamente en comparación con los valores históricos registrados desde el año 2000. El objetivo planteado para la presente investigación se centra en exponer sobre fundamentalmente sobre los aspectos generales de la cardiopatía reumática en la actualidad, así como también sobre su diagnóstico y tratamiento. Se concluye, entre otros aspectos, destacando lo trascendente que sigue siendo el diagnóstico temprano y prevención de este tipo de complicaciones potencialmente fatales, no solo para evitar su proliferación sino también para determinar el tratamiento más idóneo según las particularidades presentes en cada persona la cardiopatía reumática.

Palabras claves: Cardiopatía; Cardiovasculares; Crónicas; Infecciosas; Fatales. 


\title{
Cardiopatía reumática diagnóstico y tratamiento
}

Vol. 3, núm. 4., (2019)

Santiago Darío Freire de la A; Sofía Teresa Valdez Castro; Antonella Fanny Montenegro

Villavicencio; Lucía Andrea Jiménez Rivera

\begin{abstract}
Rheumatic heart disease is considered one of the most serious cardiovascular scourges of the last century. The disease was almost eradicated in the developed world in the 1980s, thanks to improvements in living conditions and the introduction of penicillin. Globally, cardiovascular diseases are the main cause of mortality, registering in 2013 more than 17.3 million deaths, a figure that by 2030 is believed to increase to more than 23.6 million. Regarding Ecuador, WHO has reported through a report that there has been evidence of an increase in the morbidity and mortality of chronic noncommunicable diseases, as well as showing that infectious diseases are still persistent (among those, evidently figured heart disease rheumatic), however, through another previous report they had estimated that by 2025 the tendency for premature death due to cardiovascular diseases would decrease significantly compared to the historical values recorded since 2000 . The objective set for the present investigation is focused on exposing fundamentally about the general aspects of rheumatic heart disease today, as well as its diagnosis and treatment. It is concluded, among other aspects, highlighting the importance of the early diagnosis and prevention of this type of potentially fatal complications, not only to prevent its proliferation but also to determine the most suitable treatment according to the particularities present in each person rheumatic heart disease.
\end{abstract}

Key words: Heart Disease; Cardiovascular; Chronic; Infectious; Fatal. 


\section{Cardiopatía reumática diagnóstico y tratamiento}

Vol. 3, núm. 4., (2019)

Santiago Darío Freire de la A; Sofía Teresa Valdez Castro; Antonella Fanny Montenegro Villavicencio; Lucía Andrea Jiménez Rivera

\section{Introducción.}

"La enfermedad cardiovascular es la causa principal de mortalidad a nivel mundial, y en 2013 causó más de 17.3 millones de muertes, una cifra que se proyecta aumentará a más de 23.6 millones para el año 2030" (American Heart Association, 2017)

Watkins et al. (2017) quienes desarrollaron una destacada investigación enfocada en estimar la carga global, regional y nacional de enfermedad cardíaca reumática en el periodo de 25 años (desde 1990 hasta 2015), entre sus conclusiones destacaron que, en todo el mundo, la evidencia demostró que carga de enfermedad cardíaca reumática relacionada con la salud, pero persisten altas tasas de enfermedad en algunas de las regiones más pobres del mundo. Así mismo destacaron que "la OMS y la Federación Mundial del Corazón han encomendado una reducción del $25 \%$ en la mortalidad por causas cardiovasculares, incluida la enfermedad cardíaca reumática, para el año 2025" (Watkins et al.; 2017)

En relación al estudio anterior, Marijon, Celermajer, \& Jouven (2017) expresaron que "La cardiopatía reumática se ubica como uno de los flagelos cardiovasculares más graves del siglo pasado. Como resultado de las mejoras en las condiciones de vida y la introducción de la penicilina, la enfermedad fue casi erradicada en el mundo desarrollado en la década de 1980"

De conformidad con el Perfil De Enfermedades Cardiovasculares - Ecuador ofrecido por la OMS (2014), para 2025 se estima que la tendencia por muerte prematura por enfermedades cardiovasculares disminuya significativamente en comparación con los valores históricos desde el año 2000, sin embargo, mediante otro tipo de reporte la OMS (2018) ha evidenciado un incremento 


\section{Cardiopatía reumática diagnóstico y tratamiento}

Vol. 3, núm. 4., (2019)

Santiago Darío Freire de la A; Sofía Teresa Valdez Castro; Antonella Fanny Montenegro

Villavicencio; Lucía Andrea Jiménez Rivera

en la morbilidad y mortalidad por las enfermedades crónicas no transmisibles, así como también se deja ver que las enfermedades infecciosas aún son persistentes; entre las que figura la cardiovascular.

El objetivo planteado para la presente investigación se centra en exponer sobre fundamentalmente sobre los aspectos generales de la cardiopatía reumática en la actualidad, así como también sobre su diagnóstico y tratamiento, valiéndonos para ello del material bibliográfico con contenido científico académico reciente que coadyuve tanto en la comprensión de la enfermedad, como bibliografía referenciable de otros estudios investigativos.

\section{Materiales y Métodos}

En base a un diseño bibliográfico, se desarrolla una metodología de revisión sistemática enfocada en la búsqueda, análisis e interpretación de literatura científico académica disponible en determinadas bases de datos, entre las que figuran: MedlinePlus, PubMed, Biblioteca Virtual de la Salud (BVS), SciELO, Dialnet, Cochrane, entre otras.

Se inicia con una búsqueda aleatoria y consecutiva en las mencionadas bases de datos, usando las expresiones "cardiopatía reumática", "fiebre reumática" y "diagnóstico y tratamiento de cardiopatías reumáticas". Los resultados de las búsquedas en conjunto sumaron más de un centenar de miles de registros bibliográficos de diversa índole, aproximadamente, que en seguida se fueron filtrando básicamente en función de los siguientes criterios: pertinencia y correlatividad temática, idioma español e inglés, publicación en los últimos siete años, sin descartar por tipo de material bibliográfico; es decir, se escogen títulos primordialmente de artículos científicos 


\section{Cardiopatía reumática diagnóstico y tratamiento}

Vol. 3, núm. 4., (2019)

Santiago Darío Freire de la A; Sofía Teresa Valdez Castro; Antonella Fanny Montenegro Villavicencio; Lucía Andrea Jiménez Rivera

originales, revisiones sistemáticas, protocolos, libros, boletines, folletos, tesis de posgrado y doctorado, noticias científicas, entre otros documentos e información de interés científico y académico.

Es a partir de allí que definitivamente, en base a un cúmulo de aproximadamente 60 contenidos bibliográficos diversos, se efectúa el análisis, interpretación y discusión de dicho material tratado como evidencia científico académica, proceso que por cierto sirvió para que, consecutiva y consensuadamente, se determinara el criterio grupal de las ideas y planteamientos aquí expuestos.

\section{Resultados}

En el Instituto del corazón de Texas de Estados Unidos de América, la fiebre reumática es igualmente concebida como la complicación de una amigdalitis estreptocócica no tratada, en donde ésta última a su vez es causada por una infección por estreptococos del grupo A presente en la garganta. La institución afirma que ésta patología “puede dañar los tejidos del organismo causando hinchazón, aunque el mayor peligro de la enfermedad es el daño que puede ocasionar al corazón”. Refieren que, en no menos del 50\% de las veces, esta complicación "produce una cicatrización de las válvulas del corazón, particularidad que puede estrechar la válvula y hacer que sea más difícil que ésta se abra bien o se cierre por completo". Otro efecto paralelo es que "el corazón tiene que esforzarse más por bombear sangre al resto del organismo”. Es entonces como el referido daño valvular puede generar lo que se define como «cardiopatía reumática» condición que a posteriori “puede ocasionar una insuficiencia cardíaca congestiva”. (Texas Heart Institute, s.f.) 


\section{Cardiopatía reumática diagnóstico y tratamiento}

Vol. 3, núm. 4., (2019)

Santiago Darío Freire de la A; Sofía Teresa Valdez Castro; Antonella Fanny Montenegro

Villavicencio; Lucía Andrea Jiménez Rivera

Es un tipo de bacteria entre varias existentes de su mismo tipo, resaltando solamente dos de ellos, los del "Grupo A" y los del "Grupo B", por causar la mayoría de infecciones en las personas. Las del "A", se asocian a infecciones en la garganta, escarlatina, impétigo, síndrome del shock tóxico, celulitis y fascitis necrotizante. Los del "B" pueden causar en los recién nacidos, infecciones de la sangre, neumonía y meningitis, sin embargo, la infección también la puede contraer un adulto, siendo más vulnerables aquellos con edades mayor a los 65 años o si padecen algún otro problema de salud, causándoles infecciones del tracto urinario, la sangre y la piel; y neumonía. (MedlinePlus, 2019)

De la misma manera, Mayo Clinic (2017) refiere que esta enfermedad inflamatoria, frecuente en niños de 5 a 15 años, es contraída por no haber tratado adecuadamente una faringitis estreptocócica o fiebre escarlatina complicadas, causadas por una infección con la bacteria estreptocócica; sin embargo, eso no significa que un adulto o niño de menos edad que los antes referido, puedan igualmente contraerla.

Agregan que esta complicación puede causar daños cardíacos permanentes, "incluidos el daño en las válvulas cardíacas y la insuficiencia cardíaca", no obstante, su tratamiento facilita potencialmente la reducción de dichos daños causados por la inflamación, alivian el dolor y los otros síntomas, aparte de que previene la reaparición esta patología.

La cardiopatía reumática está causada por la lesión de las válvulas cardiacas y el miocardio derivada de la inflamación y la deformación cicatrizal ocasionada por la fiebre reumática. Es generada por una respuesta anormal del organismo ante una infección con bacterias 


\section{Cardiopatía reumática diagnóstico y tratamiento}

Vol. 3, núm. 4., (2019)

Santiago Darío Freire de la A; Sofía Teresa Valdez Castro; Antonella Fanny Montenegro Villavicencio; Lucía Andrea Jiménez Rivera

estreptocócicas, que suele manifestarse en forma de dolor de garganta o amigdalitis en los niños. (Gutiérrez, 2015)

"La cardiopatía reumática es una causa importante de enfermedad cardíaca global y ocurre luego de uno o más episodios de fiebre reumática, una condición asociada con una respuesta inmune inapropiada a la infección por el Estreptococo del Grupo A.” (Schroh, 2019)

La misma autora continúa indicando que en la cardiopatía reumática las válvulas más comúnmente afectadas son; las válvulas mitrales y aórticas.

Estenosis de la válvula mitral.

Referido a un "estrechamiento de la apertura de la válvula mitral, aumentando la resistencia del flujo de la sangre desde el atrio izquierdo hasta el ventrículo izquierdo.” (GW Heart \& Vascular Institute, 2019)

Estenosis de la válvula aortica.

También conocida como estenosis aórtica, se produce cuando la válvula aórtica del corazón se estrecha. Este estrechamiento impide que la válvula se abra por completo, lo que reduce u obstruye el flujo sanguíneo del corazón a la arteria principal del cuerpo (aorta) y hacia el resto del organismo. Cuando el flujo de sangre que pasa por la válvula aórtica se reduce o se obstruye, el corazón debe trabajar más para bombear sangre al cuerpo. Con el tiempo, este esfuerzo adicional limita la cantidad de sangre que puede bombear el corazón, lo que puede provocar síntomas y, posiblemente, debilitar el músculo cardíaco. (Mayo Clinic, 2018) 


\section{Cardiopatía reumática diagnóstico y tratamiento}

Vol. 3, núm. 4., (2019)

Santiago Darío Freire de la A; Sofía Teresa Valdez Castro; Antonella Fanny Montenegro

Villavicencio; Lucía Andrea Jiménez Rivera

\section{Insuficiencia mitral}

Es la incompetencia de la válvula mitral que moviliza el flujo desde el ventrículo izquierdo hacia la aurícula izquierda durante la sístole ventricular. La insuficiencia mitral puede ser primaria (son causas frecuentes el prolapso de la válvula mitral y la fiebre reumática) o secundaria a dilatación del ventrículo izquierdo o infarto.(Armstrong, 2016)

\section{Insuficiencia aortica}

Se produce por el cierre defectuoso de la válvula aórtica que genera una fuga (regurgitación) de sangre desde la aorta hacia el ventrículo izquierdo en cada latido. Esta fuga de sangre, genera una sobre carga de volumen en el ventrículo izquierdo que con el tiempo puede dilatarse. (Clínica Universitaria de Navarra, 2019)

\section{Diagnóstico}

Primero se realiza un cultivo de la garganta para detectar la presencia de una infección estreptocócica. Para realizar el análisis de amigdalitis estreptocócica, el médico pasa un hisopo por el fondo de la garganta. Las bacterias recogidas con el hisopo se colocan en lo que se denomina un «medio de cultivo», donde las bacterias pueden crecer para poder ser analizadas.

A continuación, el médico emplea un estetoscopio para auscultar el corazón. También buscará nódulos en las articulaciones. A veces puede ser necesario realizar análisis de sangre, radiografías de tórax o un electrocardiograma (ECG) para obtener un diagnóstico más preciso. (Texas Heart Institute, s.f.) 


\section{Cardiopatía reumática diagnóstico y tratamiento}

Vol. 3, núm. 4., (2019)

Santiago Darío Freire de la A; Sofía Teresa Valdez Castro; Antonella Fanny Montenegro Villavicencio; Lucía Andrea Jiménez Rivera

Por su parte, el Centro de Salud Infantil de Stanford (2019) indica que para el diagnóstico de la enfermedad cardíaca reumática, inicialmente el médico tratante debe informarse sobre los síntomas del paciente y su historia clínica, incluida la ocurrencia de infecciones por estreptococos o fiebre reumática, así como también debe efectuar un examen físico y, posiblemente otros análisis y pruebas, tales como:

- Electrocardiografía; que se trata de una prueba para medir la actividad eléctrica del corazón.

- Ecocardiografía: referida a una prueba que, mediante el uso de ondas sonoras (ultrasonido), produce imágenes detalladas del corazón.

- Análisis de sangre. (Centro de Salud Infantil de Stanford, 2019)

\section{Tratamiento}

En niños.

En principio, éste dependerá de los síntomas, la edad y el estado general de salud del enfermo, y a su vez variará en relación la gravedad de la afección. El paciente probablemente será derivado a cardiología pediátrica, y en base a su sintomatología, consecutivamente se referirá a otras especialidades médicas. El reposo será necesario particularmente en los pacientes pediátricos con esta complicación, hasta que mejoren sus síntomas. Además, el cardiólogo pediatra podría indicar cualquiera de estos medicamentos, solos o combinados:

- Antibióticos; para tratar la infección. 


\section{Cardiopatía reumática diagnóstico y tratamiento}

Vol. 3, núm. 4., (2019)

Santiago Darío Freire de la A; Sofía Teresa Valdez Castro; Antonella Fanny Montenegro

Villavicencio; Lucía Andrea Jiménez Rivera

- Medicamentos esteroideos o AINEs; para el alivio de la inflamación del corazón y otras partes del cuerpo.

- Pastillas contra la retención de líquidos (diuréticos) para la insuficiencia cardíaca.

- Otros medicamentos.

La cirugía será necesaria sólo en algunos casos, para corregir o reemplazar las válvulas dañadas del corazón. (Centro de Salúd Infantil de Stanford, 2019)

Por su parte, (Mayo Clinic, 2017) destaca que, teniendo como base que el principalmente el objetivo vendría siendo el de destruir las bacterias estreptocócicas del grupo A restantes, aliviar los síntomas, controlar la inflamación y prevenir las recurrencias, los tratamientos a indicar serían:

$\checkmark$ Antibióticos en dos ciclos; para eliminar las bacterias estreptocócicas restantes y prevenir la recurrencia de la fiebre reumática. Este tratamiento preventivo, es probable que continúe hasta los 21 años o hasta que el paciente complete un curso mínimo de cinco años de tratamiento, o lo que sea más largo. Es recomendable para las personas diagnosticadas con inflamación cardíaca durante la fiebre reumática que tomen el tratamiento antibiótico preventivo durante 10 años o más.

$\checkmark$ Tratamiento antiinflamatorio; con analgésico tales como aspirina o naproxeno, para reducir la inflamación, la fiebre y el dolor. Si los síntomas son graves o si el paciente no responde a los medicamentos antiinflamatorios, se receta un corticosteroide.

$\checkmark$ Medicamentos anticonvulsivos; entre los que figuran ácido valproico (Depakene) o carbamazepina (Carbatrol, Tegretol, otros), para prevenir los fuertes movimientos involuntarios causados por la Corea de Sydenham. 


\section{Cardiopatía reumática diagnóstico y tratamiento}

Vol. 3, núm. 4., (2019)

Santiago Darío Freire de la A; Sofía Teresa Valdez Castro; Antonella Fanny Montenegro Villavicencio; Lucía Andrea Jiménez Rivera

\section{En Adultos.}

Estará basado en: el estado general de salud y la historia médica, que tan avanzada está la enfermedad, la tolerancia a determinados medicamentos, procedimientos o terapias, expectativas para la trayectoria de la enfermedad, la opinión o preferencia. (GW Heart \& Vascular Institute, 2019)

Villa-Forte \& Mandell (2012) entre otras cosas destacaron que "las manifestaciones de la enfermedad cardiaca en estos pacientes van de subclínicas a graves y pueden requerir un tratamiento inmunosupresor agresivo. La detección temprana es importante para instaurar con rapidez un tratamiento apropiado". Además, para estos casos, dichos tratamientos se hacen en base en la gravedad; requiriendo frecuentemente un tratamiento combinado con corticoides y fármacos citotóxicos en los casos más graves.

\section{Conclusión}

Partiendo nuevamente del hecho de que esta complicación es plenamente previsible, es imprescindible destacar que lo trascendente que sigue siendo el diagnóstico temprano y prevención de este tipo de complicaciones potencialmente fatales, no solo para evitar su proliferación sino también para determinar el tratamiento más idóneo según las particularidades presentes en cada persona la cardiopatía reumática.

Sin embargo, se considera igualmente valioso mencionar algunos aspectos que, en la reciente 71a Asamblea Mundial de la Salud sobre la fiebre reumática y cardiopatía reumática, se han destacado. En la reunión, entre otras cosas, se dejó constancia de que si bien es inquietante 


\section{Cardiopatía reumática diagnóstico y tratamiento}

Vol. 3, núm. 4., (2019)

Santiago Darío Freire de la A; Sofía Teresa Valdez Castro; Antonella Fanny Montenegro Villavicencio; Lucía Andrea Jiménez Rivera

tener presente “[...] que la cardiopatía reumática es una causa importante y prevenible de morbilidad y mortalidad en todas las regiones de la OMS", es reconocido que ser su temprano diagnóstico favorezca la reducción de "la morbilidad y la mortalidad de forma costo eficaz" manera considerable. (Asamblea Mundial de la Salud, 71, 2018)

Por lo tanto, retomando lo expresado por la directora de la Organización Panamericana de la Salud (OPS) respecto a las enfermedades no transmisibles (entre las que obviamente figura la cardiopatía reumática) dijo "son la plaga de nuestro tiempo", enfatizando que "se debe mantener un mensaje simple, efectivo e invertir más en su comunicación” de dichas enfermedades. En definitiva, culmina alegando que "si queremos ser exitosos en revertir la catástrofe que están causando las ENT necesitamos un movimiento social masivo, nada menos" (OPS, 2019)

\section{Bibliografía}

American Heart Association. (25 de enero de 2017). professional.heart.org. (C. d. Association, Ed.) doi:10.1161/CIR.0000000000000485.

Armstrong, G. (Septembre de 2016). msdmanuals.com. Recuperado el 25 de 07 de 2019, de msdmanuals.com: $\quad$ https://www.msdmanuals.com/es-ve/professional/trastornoscardiovasculares/enfermedades-valvulares/insuficiencia-

mitral?query=Insuficiencia $\% 20 \mathrm{mitral} \% 20$ secundaria

Asamblea Mundial de la Salud, 71. (2018). 71. a Asamblea Mundial de la Salud. Fiebre reumática y cardiopatía reumática (pág. 3 p.). Ginebra: WHO. Recuperado el 26 de 07 de 2019, de https://apps.who.int/iris/handle/10665/279548

Centro de Salúd Infantil de Stanford. (2019). stanfordchildrens.org/es. (Stanford Children's Health) Recuperado el 26 de 07 de 2019, de stanfordchildrens.org/es: https://www.stanfordchildrens.org/es/topic/default?id=cardiopatareumtica-90-P04919

Clínica Universitaria de Navarra. (2019). cun.es. Recuperado el 26 de 07 de 2019, de cun.es: https://www.cun.es/enfermedades-tratamientos/enfermedades/insuficiencia-aortica 


\section{Cardiopatía reumática diagnóstico y tratamiento}

Vol. 3, núm. 4., (2019)

Santiago Darío Freire de la A; Sofía Teresa Valdez Castro; Antonella Fanny Montenegro Villavicencio; Lucía Andrea Jiménez Rivera

Gutiérrez, J. (15 de Noviembre de 2015). Cardiopatía reumática. Diario Correo del Sur, pág. 1 p. Recuperado el 26 de 04 de 2019, de https://correodelsur.com: https://correodelsur.com/ecos/20151115_cardiopatia-reumatica.html

GW Heart \& Vascular Institute. (2019). gwheartandvascular.org. Recuperado el 26 de 07 de 2019, de gwheartandvascular.org: http://www.gwheartandvascular.org/education/enespanol/enfermedades/enfermedades_condiciones/enfermedad-reumatica-del-corazon/

GW Heart \& Vascular Institute. (2019). gwheartandvascular.org. Recuperado el 27 de 07 de 2019, de gwheartandvascular.org: http://www.gwheartandvascular.org/education/enespanol/enfermedades/enfermedades_condiciones/enfermedades_condiciones_valvulares/

Marijon, E., Celermajer, D., \& Jouven, X. (2017). Rheumatic Heart Disease - An Iceberg in Tropical Waters. New England Journal Medicine, 377(8), 780-781. doi:10.1056/NEJMe1705840

Mayo Clinic. (17 de Noviembre de 2017). mayoclinic.org. Recuperado el 25 de 07 de 2019, de https://www.mayoclinic.org/diseases-conditions/rheumatic-fever/diagnosis-treatment/drc20354594

Mayo Clinic. (17 de Noviembre de 2017). www.mayoclinic.org. Recuperado el 26 de 07 de 2019, de www.mayoclinic.org: https://www.mayoclinic.org/es-es/diseasesconditions/rheumatic-fever/symptoms-causes/syc-20354588

Mayo Clinic. (21 de Septiembre de 2018). mayoclinic.org. Recuperado el 25 de 07 de 2019, de mayoclinic.org: https://www.mayoclinic.org/es-es/diseases-conditions/aorticstenosis/symptoms-causes/syc-20353139

MedlinePlus. (2019). medlineplus.gov/spanish. Recuperado el 25 de 07 de 2019, de medlineplus.gov/spanish: https://medlineplus.gov/spanish/streptococcalinfections.html

OMS. (2014). https://www.paho.org. Recuperado el 27 de 07 de 2019, de https://www.paho.org: https://www.paho.org/hq/dmdocuments/2014/ECUADOR-PERFIL-ECV-2014.pdf

OMS. (Mayo de 2018). who.int/iris. Recuperado el 26 de 07 de 2019, de https://apps.who.int/iris/bitstream/handle/10665/137163/ccsbrief_ecu_en.pdf?sequence=1

OPS. (2019). Comunicar efectivamente sobre las enfermedades no transmisibles es un gran desafío. Organización Panamericana de Salud. Washington, D.C.: Oficina Regional para las Américas de la Organización Mundial de la Salud. Recuperado el 26 de 07 de 2019, de https://www.paho.org/hq/index.php?option=com_content $\& v i e w=\operatorname{article} \& i d=15171$ :comu nicar-efectivamente-sobre-las-enfermedades-no-transmisibles-es-un-grandesafio\&Itemid=72512\&lang=es 


\section{Cardiopatía reumática diagnóstico y tratamiento}

Vol. 3, núm. 4., (2019)

Santiago Darío Freire de la A; Sofía Teresa Valdez Castro; Antonella Fanny Montenegro

Villavicencio; Lucía Andrea Jiménez Rivera

Schroh, A. (11 de abril de 2019). siacardio.com. Recuperado el 25 de 07 de 2019, de siacardio.com: http://www.siacardio.com/consejos/enfermedad-reumatica/manejo-medico-de-lacardiopatia-reumatica/

Texas Heart Institute. (s.f.). https://www.texasheart.org. Recuperado el 25 de 07 de 2019, de https://www.texasheart.org: https://www.texasheart.org/heart-health/heart-informationcenter/topics/fiebre-reumatica/

Watkins, D., Johnson, C., Colquhoun, S., Karthikeyan, G., Beaton, A., Bukhman, G., . . . al., e. (24 de Agosto de 2017). Global, Regional, and National Burden of Rheumatic Heart Disease, 1990-2015. New England Journal of Medicine, 377(8), 713-722. doi:10.1056/NEJMoa1603693

$$
\text { (2) }(\mathbb{D} \otimes(0)
$$

RECONOCIMIENTO-NOCOMERCIAL-COMPARTIRIGUAL

CC BY-NC-SA

ESTA LICENCIA PERMITE A OTROS ENTREMEZCLAR, AJUSTAR Y CONSTRUIR A PARTIR DE SU OBRA CON FINES NO COMERCIALES, SIEMPRE Y CUANDO LE RECONOZCAN LA AUTORÍA Y SUS NUEVAS CREACIONES ESTÉN BAJO UNA LICENCIA CON LOS MISMOS TÉRMINOS. 